\title{
Texture classification using invariant ranklet features
}

\author{
Matteo Masotti*, Renato Campanini \\ Department of Physics, University of Bologna \\ Viale Berti-Pichat 6/2, 40127, Bologna, Italy
}

\begin{abstract}
A novel invariant texture classification method is proposed. Invariance to linear/nonlinear monotonic gray-scale transformations is achieved by submitting the image under study to the ranklet transform, an image processing technique relying on the analysis of the relative rank of pixels rather than on their gray-scale value. Some texture features are then extracted from the ranklet images resulting from the application at different resolutions and orientations of the ranklet transform to the image. Invariance to $90^{\circ}$-rotations is achieved by averaging, for each resolution, correspondent vertical, horizontal, and diagonal texture features. Finally, a texture class membership is assigned to the texture feature vector by using a support vector machine (SVM) classifier. Compared to three recent methods found in literature and having being evaluated on the same Brodatz and Vistex datasets, the proposed method performs better. Also, invariance to linear/non-linear monotonic gray-scale transformations and $90^{\circ}$-rotations are evidenced by training the SVM classifier on texture feature vectors formed from the original images, then testing it on texture feature vectors formed from contrast-enhanced, gamma-corrected, histogram-equalized and $90^{\circ}$-rotated images.
\end{abstract}

Key words: Ranklets, Support Vector Machine, Texture, Brodatz, VisTex

\footnotetext{
* Corresponding author. Tel: +39 051 2095136. Fax: +39 051 2095047. E-mail: masotti@bo.infn.it. Web: http://www.bo.infn.it/ masotti/
} 


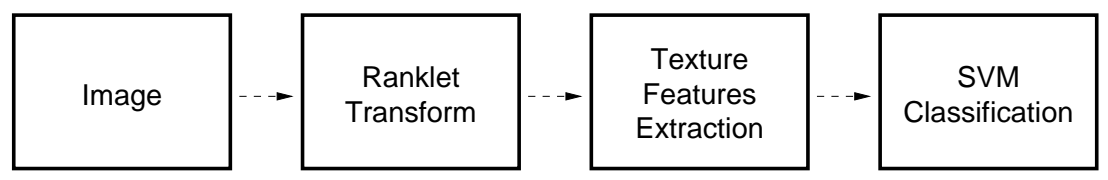

Fig. 1. Block diagram of the proposed approach.

\section{Introduction}

Texture is a surface property which gives a combined information on the smoothness, coarseness, and regularity of objects (Gonzalez and Woods, 1992). On digital images, it reflects as local variations of the gray-scale content.

In the last years, owing to the increasing spread of digital image databases, automatic texture classification has started playing a key role, with several applications in biomedical imaging, remote sensing, image classification and segmentation. The typical automatic texture classification system involves two steps: (1) a feature extraction step, where a set of texture features are extracted from the image under study and (2) a classification step, where a texture class membership is assigned to it according to the extracted texture features. The majority of the existing systems assume that the images to be classified are identical to those used in training, for instance, with respect to gray-scale range and rotation. Nevertheless, this is a strong assumption, as for the most part of practical applications it is very unlikely that images are captured under the same conditions of illumination and viewpoint. As a result, in the last years, great attention has been devoted to invariant automatic texture classification. Systems having robustness to linear monotonic variations of the gray-scale were proposed by Partio et al. (2004) and Sukanya (2000). Rotation invariance texture classification was addressed in many several ways: Chetverikov (1982) used texture anisotropy, Davis et al. (1979) used generalized co-occurrence matrices, Pichler et al. (1996) and Idrissa and Acheroy (2002) used banks of Gabor filters, whereas Charalampidis and Kasparis (2002) and Manthalkar et al. (2003b) used wavelet-based filters. Systems which incorporate invariance to both linear monotonic variations of the grayscale and rotation are much more rare in literature. Two of the earliest ones are those proposed by Chen and Kundu (1994) and Wu and Wei (1996), where hidden Markov models were used. More recently, Ojala et al. (2002) proposed a system based on local binary patterns.

This work proposes a system invariant to linear/non-linear monotonic grayscale transformations and $90^{\circ}$-rotations based on a novel image processing technique, known as ranklet transform, in combination with support vector machine (SVM) classification. A schematic block diagram of this system is shown in Fig. 1. First, given an image $I$, the ranklet transform is applied (Smeraldi, 2002). As discussed in some of our recent works (Masotti, 2006a; Campanini et al., 2006; Masotti, 2006b), in fact, being calculated from the relative rank of pixels rather than from their gray-scale value (i.e., non-parametric property of the ranklet transform), this transform allows to produce a gray- 
scale invariant image representation; more specifically, as it will be shown in the following, gray-scale invariance is intended as to linear/non-linear monotonic gray-scale transformations of the original image $I$, e.g., brigthness variation, contrast enhancement, gamma correction, histogram equalization (Gonzalez and Woods, 1992). Also, being calculated as a multi-resolution and orientation-selective analysis (i.e., multi-resolution and orientation-selective properties of the ranklet transform), this transform allows to recognize analogous characteristics at different resolutions and orientations of the image as well. From the ranklet images $R I$ which result from the application of the ranklet transform to the image $I$, up to 11 texture features similar to those described by Xu et al. (2006) are hence extracted. Then, by means of an SVM classifier (Vapnik, 1995, 1998), a texture class membership is assigned to the texture feature vector resulting from the processing of the image $I$.

This system is evaluated on four different image datasets extracted from the VisTex and Brodatz albums (MIT Media Lab, 1995; Brodatz, 1966). Specifically, invariance to linear/non-linear monotonic gray-scale transformations is investigated by first training SVM on texture feature vectors formed from original images, then testing it on texture feature vectors formed from (different) contrast-enhanced, gamma-corrected, and histogram equalized-images: contrast enhancement is a gray-scale transformation which maps linearly the gray-scale values of the processed image to a new range; gamma correction is a gray-scale transformation whose mapping is non-linear, as weighted toward brighter or darker gray-scale values; image equalization, instead, is a non-linear gray-scale transformation which spreads the gray-scale values of the processed image evenly distributed over the entire range (Gonzalez and Woods, 1992). As far as $90^{\circ}$-rotation invariance, SVM is trained on texture feature vectors formed from original images, then tested on texture feature vectors formed from (different) $90^{\circ}-, 180^{\circ}$-, and $270^{\circ}$-rotated images. The rationale behind the decision of evaluating the proposed automatic texture classification system on standard benchmark images as those in the Vistex and Brodatz albums is twofold. First, to propose a system as general-purpose as possible, rather than overly suited for a specific texture classification problem. Second, to be able to compare the proposed system directly with three recent automatic texture classification systems found in literature and having being evaluated on the same images, namely, (1) a texture classification approach based on ridgelets and minimum distance classification (Arivazhagan et al., 2006), (2) a rotation-invariant approach based on wavelets and minimum distance classification (Muneeswaran et al., 2005), and (3) an approach based on wavelets and SVM (Li et al., 2003).

\section{Ranklet transform}

The ranklet transform of an image $I$ involves three phases: (1) multi-resolution, (2) orientation-selective, and (3) non-parametric analysis (Smeraldi, 2002). As 


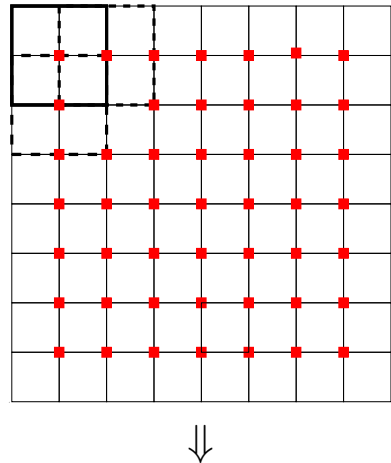

Res $2=49$ crops

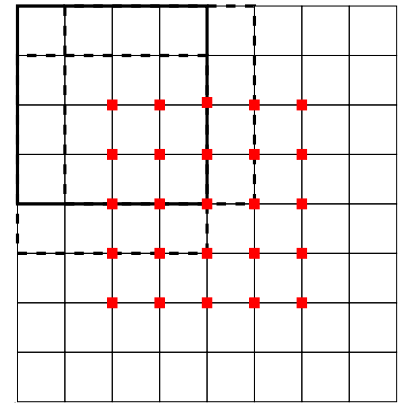

$\Downarrow$

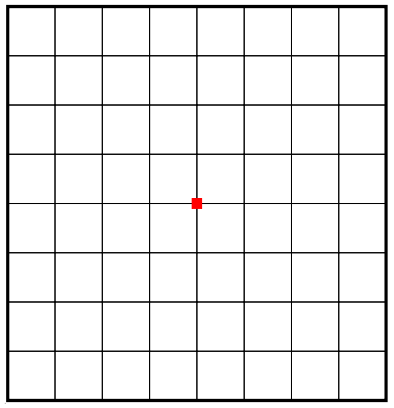

$\Downarrow$

Res $4=25$ crops

Fig. 2. Ranklet transform, multi-resolution analysis. At resolutions $\{2,4,8\}$, up to 49,25 , and 1 overlapping square crops are extracted from an example image with size $8 \times 8$.

a result of the application of the ranklet transform to an image $I$, a number $n_{R I}$ of non-parametric ranklet images $R I$ are produced, this number being equal to the number $n_{R}$ of resolutions at which the analysis is performed times the number $n_{O}$ of orientations considered, i.e., $n_{R I}=n_{R} \times n_{O}$.

Multi-resolution analysis is achieved by choosing a set of resolutions, then extracting from the image $I$ a variable number of overlapping square crops having these resolutions as linear size. For example, see Fig. 2, given an image $I$ of size $8 \times 8$, the analysis at resolutions $\{2,4,8\}$ results in 49,25 , and, 1 square crops having linear size 2,4 , and 8 , respectively. In general, for each resolution, the number of extracted square crops is computed as $\left(l_{I}+1-l_{C}\right)^{2}$, where $l_{I}$ represents the linear size of the image $I$ and $l_{C}$ that of the square crops.

For each resolution, orientation-selective analysis is then achieved by splitting half of the $N$ pixels of each square crop into a subset $T$ and the remaining half into a subset $\mathrm{C}$, these pairs of subsets being defined differently depending on the orientation considered. In particular, as shown in Fig. 3, the two subsets $T$ and $C$ are defined differently for three orientations, namely, as $T_{V}$ and $C_{V}$ for the vertical orientation, $T_{H}$ and $C_{H}$ for the horizontal orientation, and $T_{D}$
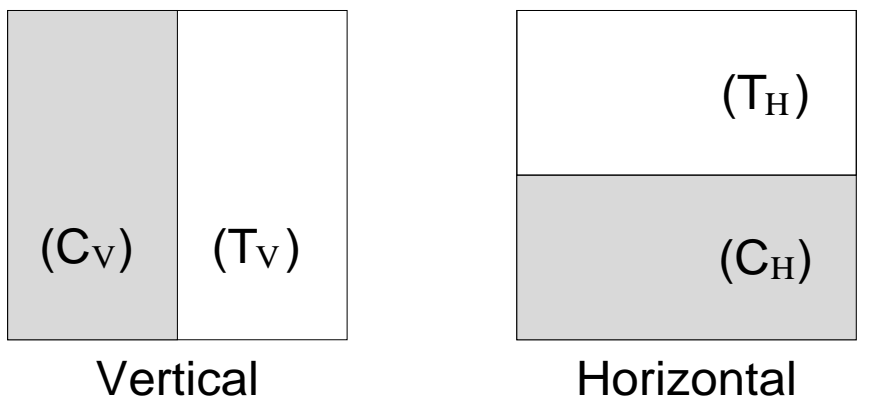

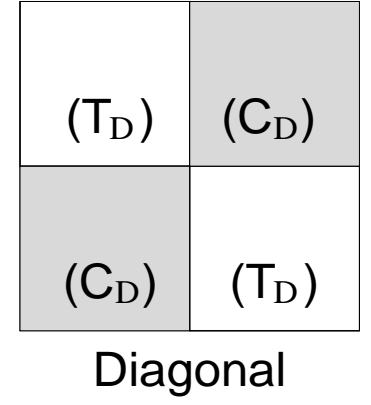

Fig. 3. Ranklet transform, orientation-selective analysis. From left to right, the vertical, horizontal, and diagonal pairs of subsets. 
and $C_{D}$ for the diagonal orientation; these subsets are evidently reminiscent of the Haar basis functions introduced by Mallat (1989) within the framework of the wavelet theory, but also fairly close to the rectangular regions adopted by Viola and Jones (2004) to compute their set of Haar-like features for real-time object detection.

Finally, for each resolution and orientation, non-parametric analysis is carried out on each square crop. By means of $N / 2 \times N / 2=N^{2} / 4$ direct comparisons, the number of pixels pairs $\left(p_{m}, p_{n}\right)$ satisfying the condition that gray-scale value of $p_{m} \in \mathrm{T}$ is higher than that of $p_{n} \in \mathrm{C}$ can be calculated; this number would naturally range from 0 to $N^{2} / 4$ but, by appropriate normalization, can be mapped into the range $[-1,+1]$. Rather than calculating the above mentioned number of pixel pairs through $O\left(N^{2}\right)$ direct comparisons, however, this is achieved in $O(N \log N)$ operations by considering the relative rank of pixels instead of their gray-scale value:

$$
R_{j}=\frac{\sum_{p \in \mathrm{T}_{j}} \pi(p)-\frac{N}{4}\left(\frac{N}{2}+1\right)}{\frac{N^{2}}{8}}-1, \quad j=\mathrm{V}, \mathrm{H}, \mathrm{D}
$$

where $\sum_{p \in \mathbf{T}_{j}} \pi(p)$ is the sum of the pixels' ranks $\pi(p)$ in $\mathbf{T}_{j}$. The derived ranklet coefficient $R_{j}$ is close to +1 as many square crop's pixels in $\mathrm{T}_{\mathrm{j}}$ have higher gray-scale value than the pixels in $C_{j}$. Conversely, it is close to -1 as many square crop's pixels in $C_{\mathrm{j}}$ have higher gray-scale value than the pixels in $\mathrm{T}_{\mathrm{j}}$. Also, $R_{V}, R_{H}$, and $R_{D}$ are close to 0 for a square crop with no vertical, horizontal, and diagonal global gray-scale value variation, respectively.

By following this approach, the ranklet decomposition of an image $I$ results for each resolution in a set of three ranklet images $R I$, i.e., vertical, horizontal, and diagonal; the pixels of each image are represented, respectively, by the ranklet coefficients $R_{V}, R_{H}$, and $R_{D}$ calculated from the square crops of that specific resolution. At a given location of one of these ranklet images, the ranklet coefficient can be thought as a measure of the regularity of its neighborhood at that specific resolution and orientation, namely, a measure of the texture; the higher its absolute value, the more variable its neighborhood. Also, for more than one resolution, the number of ranklet images is equal to the number of resolutions times the three orientations considered, i.e., $n_{R I}=n_{R} \times n_{O}=$ $n_{R} \times 3$.

\section{Texture features extraction}

For each ranklet image $R I$ derived from the ranklet decomposition at different resolutions and orientations of an image $I$, the texture features extraction step involves two phases: (1) quantization of the ranklet image and (2) extraction from the quantized ranklet image of $n_{F}$ texture features (Xu et al., 2006). Quantization is aimed at discretizing the continuous real values taken by the ranklet coefficients of the ranklet image. In this work, the discrete number of quantization bins is chosen equal to 21; as a result, the ranklet values 
$r v(i), i=1, \ldots, 21$ taken by the quantized ranklet coefficients are $(-1,-0.9$, $\ldots,-0.1,0,+0.1, \ldots,+0.9,+1)$.

From the quantized ranklet image, $n_{F}=11$ texture features are then calculated. They are based on two texture measures, i.e., the ranklet histogram $r h$ and the ranklet co-occurrence matrix $r \mathrm{~cm}_{d, \theta}$. The ranklet histogram $r h$ represents the probability distribution of the values taken by the ranklet coefficients in the quantized ranklet image. It is defined as:

$$
r h(i)=\frac{n(i)}{\sum_{j=1}^{21} n(j)}, \quad i=1, \ldots, 21
$$

where $n(i)$ is the number of ranklet coefficients in the quantized ranklet image taking value $r v(i)$. Instead, the ranklet co-occurrence matrix $r c m_{d, \theta}$ represents the probability distribution of the transitions between all pairs of two ranklet coefficients in the quantized ranklet image. It is defined as:

$$
r c m_{d, \theta}(i, j)=\frac{n_{d, \theta}(i, j)}{\sum_{l=1}^{21} \sum_{k=1}^{21} n_{d, \theta}(l, k)}, \quad i, j=1, \ldots, 21
$$

where $n_{d, \theta}(i, j)$ represents the number of two ranklet coefficients at spatial locations $(x, y)$ and $(w, z), d$-pixel apart along the angular rotation $\theta$, and such that $R I(x, y)=r v(i)$ and $R I(w, z)=r v(j)$; the normalization factor $\sum_{l=1}^{21} \sum_{k=1}^{21} n_{d, \theta}(l, k)$ represents the total number of transitions.

The first two texture features are derived from the ranklet histogram $r h$.

1 - Mean convergence

$$
m c=\sum_{i=1}^{21} \frac{1}{\sigma} \cdot|r v(i) \cdot \operatorname{rh}(i)-\mu|, \quad \begin{aligned}
\mu & =\text { ranklet coefficients' mean } \\
\sigma & =\text { ranklet coefficients' stand. deviation }
\end{aligned}
$$

2 - Code variance

$$
c v=\sum_{i=1}^{21}(r v(i)-\mu)^{2} \cdot r h(i)
$$

The remaining texture features are derived from the average of four ranklet co-occurrence matrices $\mathrm{rcm}_{d, \theta}$ corresponding to $\theta=\left(0^{\circ}, 45^{\circ}, 90^{\circ}, 135^{\circ}\right)$ and with $d$ set equal to 1, i.e., $r c m=\frac{1}{4}\left(r c m_{1,0^{\circ}}+r c m_{1,45^{\circ}}+r c m_{1,90^{\circ}}+r c m_{1,135^{\circ}}\right)$. 3 - Code entropy

$$
c e=\sum_{i=1}^{21} \sum_{j=1}^{21} r c m(i, j) \cdot \log (r c m(i, j))
$$

4-Uniformity

$$
\text { un }=\sum_{i=1}^{21} \sum_{j=1}^{21} r \mathrm{~cm}(i, j)^{2}
$$

5/6 - First-order/second-order element difference moment

$$
\begin{aligned}
& f d m=\sum_{i=1}^{21} \sum_{j=1}^{21}|i-j| \cdot \operatorname{rcm}(i, j) \\
& s d m=\sum_{i=1}^{21} \sum_{j=1}^{21}(i-j)^{2} \cdot \operatorname{rcm}(i, j)
\end{aligned}
$$


7/8 - First-order/second-order inverse element difference moment

$$
\begin{aligned}
& f i d m=\sum_{i=1}^{21} \sum_{j=1}^{21} \frac{1}{1+|i-j|} \cdot \operatorname{rcm}(i, j) \\
& \operatorname{sidm}=\sum_{i=1}^{21} \sum_{j=1}^{21} \frac{1}{1+(i-j)^{2}} \cdot \operatorname{rcm}(i, j)
\end{aligned}
$$

9/10/11 - Energy distribution of the ranklet co-occurrence matrix

$$
\begin{aligned}
& \text { edrcm } 1=\sum_{i=9}^{13} \sum_{j=9}^{13} \operatorname{rcm}(i, j) \\
& \text { edrcm } 2=\sum_{i=7}^{15} \sum_{j=7}^{15} \operatorname{rcm}(i, j)-e d c m 1 \\
& \text { edrcm } 3=\sum_{i=3}^{19} \sum_{j=3}^{19} \operatorname{rcm}(i, j)-e d c m 1-e d c m 2
\end{aligned}
$$

As a result of the described texture features extraction step, each ranklet image $R I$ is encoded by a $1 \times 11$ texture feature vector.

\section{Gray-scale and $90^{\circ}$-rotation invariance}

The way ranklet images $R I$ are obtained from the image $I$ represents the basis for the invariance to linear/non-linear monotonic gray-scale transformations and $90^{\circ}$-rotations of the proposed approach.

As ranklet coefficients are computed from the relative rank of square crops' pixels, rather than their gray-scale values, ranklet images are very robust to gray-scale variations. This is clear in Fig. 4, where correspondent ranklet images obtained by decomposing an image, its contrast enhanced version (i.e., linear monotonic gray-scale transformation), its gamma corrected version (i.e., power-law non-linear monotonic gray-scale transformation), and its histogram equalized version (i.e., non-linear monotonic gray-scale transformation) are nearly identical. As a result, any texture feature calculated from one of such ranklet images is almost completely invariant to linear/non-linear monotonic gray-scale transformations as well.

As far as $90^{\circ}$-rotation invariance, ranklet images are computed at three different orientations with the precise intention to perceive texture patterns in more than one single direction. As each ranklet image is representative of a specific orientation, any texture feature calculated from one of such ranklet images cannot be rotation invariant by itself. However, if calculated as the average value of that texture feature over the vertical, horizontal, and diagonal ranklet images, the new averaged texture feature becomes almost completely invariant for $90^{\circ}$-rotations (Porter and Canagarajah, 1997; Manthalkar et al., 2003a).

In this work, to achieve both gray-scale and $90^{\circ}$-rotation invariance, an image $I$ is hence encoded by a texture feature vector obtained by concatenating a number of $1 \times 11$ texture feature vectors equal to the number of ranklet images resulting for its ranklet decomposition, i.e., $n_{R I}=n_{R} \times n_{O}=n_{R} \times 3$. For each resolution, correspondent vertical, horizontal, and diagonal texture 


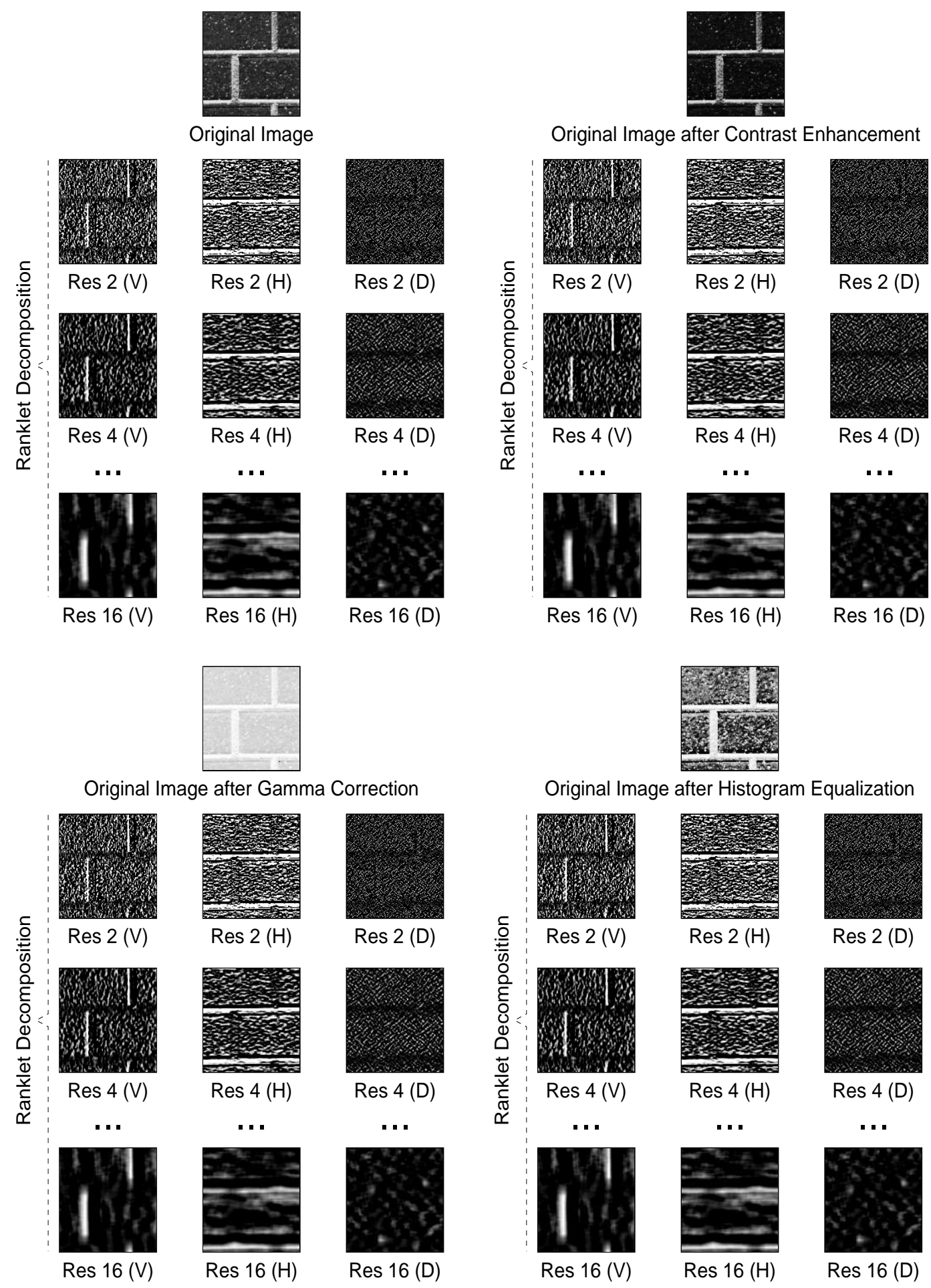

Fig. 4. Gray-scale invariance of the ranklet transform. Ranklet decomposition, from resolution 2 up to 16 , of the original image, of the original image after contrast enhancement (i.e., linear monotonic gray-scale transformation), of the original image after gamma correction (i.e., power-law non-linear monotonic gray-scale transformation), and of the original image after histogram equalization (i.e., non-linear monotonic gray-scale transformation). Regardless of the linear/non-linear gray-scale transformation, the resulting ranklet decompositions are almost identical. 
features are then averaged, thus ending up with a number of concatenated $1 \times 11$ vectors of averaged texture features equal to the number of resolutions, i.e., $n_{R}$.

\section{SVM classification}

The last step of the proposed approach consists in using SVM classification to assign a texture class membership to the texture feature vector encoding the image $I$.

In the context of two-class classification problems, SVM is a learning system that uses an optimal hyperplane to separate sets of feature vectors into two classes (Vapnik, 1995, 1998). During the training phase, the optimal hyperplane is designed as the one with the maximum margin of separation between the two classes. Once completed the training phase, during the test phase, a feature vector not used for training is then classified as belonging to either one of the two classes according to its position with respect to the optimal hyperplane.

In this work, to deal with a number of classes $k$ greater than two, the oneagainst-one approach is used, i.e., $k(k-1) / 2 \mathrm{SVM}$ classifiers are constructed being trained on feature vectors from two class memberships only, then a test feature vector is associated with the class membership to which it is more often associated by the different SVM classifiers (Weston and Watkins, 1998).

\section{Tests}

\subsection{Data}

For evaluation of the proposed approach, four different image datasets are used, i.e., Test-1, Test-2, Test-3, and Test-4. Test-1 and Test-3 are comprised of $30^{1}$ and $31^{2}$ images of size $512 \times 512$ obtained from the VisTex album (MIT Media Lab, 1995), then subdivided into 480 and 496 non-overlapping image regions of size $128 \times 128$, respectively. Test- 2 and Test- 4 are comprised of $26^{3}$

1 Bark.0006, Brick.0000, Brick.0004, Brick.0005, Clouds.0001, Fabric.0000, Fabric.0006, Fabric.0007, Fabric.0013, Fabric.0015, Fabric.0017, Fabric.0019, Flowers.0005, Flowers.0006, Food.0000, Food.0001, Leaves.0003, Leaves.0012, Metal.0000, Metal.0002, Metal.0004, Misc.0001, Misc.0002, Sand.0000, Sand.0002, Stone.0005, Tile.0004, Tile.0008, Water.0005, Wood.0002

2 Bark.0001, Bark.0004, Bark.0005, Bark.0006, Bark.0010, Brick.0000, Brick.0004, Brick.0005, Fabric.0000, Fabric.0002, Fabric.0007, Fabric.0009, Flowers.0005, Flowers.0006, Food.0001, Food.0002, Food.0004, Food.0005, Food.0006, Leaves.0002, Leaves.0003, Leaves.0004, Leaves.0008, Leaves.0010, Metal.0000, Metal.0005, Misc.0001, Sand.0002, Tile.0007, Water.0005, Wood.0002

3 D1, D3, D4, D5, D6, D8, D9, D11, D16, D17, D18, D20, D21, D25, D26, D27, D28, D29, D33, D36, D78, D87, D92, D95, D101, D102 
and $30^{4}$ images of size $640 \times 640$ obtained from the Brodatz album (Brodatz, 1966), then subdivided into 650 and 750 non-overlapping image regions of size $128 \times 128$, respectively. In order to verify the invariance to linear/nonlinear monotonic gray-scale transformations of the proposed texture features, a transformed version of the original 30,31, 26, and 30 images is also produced by contrast enhancing, gamma correcting and histogram equalizing each one of them, then extracting non-overlapping image regions as described above. As far as $90^{\circ}$-rotation invariance, four rotated versions are produced by rotating each one of the original images by $0^{\circ}, 90^{\circ}, 180^{\circ}$, and $270^{\circ}$, then again extracting non-overlapping image regions.

Each image region is submitted to the ranklet transform and decomposed into $n_{R}=7$ ranklet resolutions (i.e., $\left.\{4,6,8,10,12,14,26\}\right)$ and $n_{O}=3$ orientations (i.e., vertical, horizontal, and diagonal). Similarly to what discussed in one of our previous works (Masotti, 2006b), this choice is as arbitrary as reasonable, since it spans over a large range of resolutions, from fine ones (encoding closeview texture details) to coarse ones (encoding broad-view texture details). In particular, preliminary tests confirmed the validity of the selected ranklet resolutions. Each image region results hence in a $1 \times 231$ texture feature vector invariant to linear/non-linear monotonic gray-scale transformations, namely, a $1 \times 11$ texture feature vector for each one of the 7 resolutions and 3 orientations. To achieve $90^{\circ}$-rotation invariance, correspondent vertical, horizontal, and diagonal texture features are averaged for each resolution, thus ending up with a $1 \times 77$ texture feature vector, namely, a $1 \times 11$ vector of texture features averaged over 3 resolutions for each one of the 7 resolutions.

\subsection{Results}

The results achieved for the 480, 650, 496, and 750 image regions of Test-1, Test-2, Test-3, and Test-4 are reported in Tab. 1. Each table's entry referring to the proposed approach (i.e., Ranklets+SVM) specifies the leave-one-out (LOO) percentage classification accuracy reached on the image regions of the dataset considered. In other words, for each dataset, SVM is trained on the whole amount of image regions, except the one used for test; by changing the test image region in a round-robin manner, training and test are then repeated a number of times equal to the number of image regions (Efron and Tibshirani, 1994). The LOO percentage classification accuracy is hence measured as $\left(n_{I R C C} \cdot 100 \%\right) / n_{I R}$, where $n_{I R C C}$ represents the number of image regions correctly classified, whereas $n_{I R}$ the number of image regions extracted from each original image. As far as the SVM kernel parameters, an RBF kernel with $\gamma=1$ and $C=100$ is chosen after tuning on Test-1. The table's columns labeled with $\mathrm{O}$ refer to the case in which the LOO training and testing phases are both carried out on image regions extracted from the original images; the columns

4 D1, D3, D6, D11, D16, D17, D20, D21, D24, D28, D29, D32, D34, D35, D46, D47, D49, D51, D52, D53, D55, D56, D57, D65, D78, D82, D84, D85, D101, D104 
Table 1

Results comparison. Ranklets+SVM represents the proposed ranklet-based approach. Ridgelets+Dist (Arivazhagan et al., 2006), Wavelets+Dist (Muneeswaran et al., 2005), and Wavelets+SVM (Li et al., 2003) refer to three different approaches evaluated on the same 30 VisTex images used in Test-1, 26 Brodatz plus 31 VisTex images used in Test-2/Test-3, and 30 Brodatz images used in Test-4, respectively. $\mathrm{O}=$ original image regions. $\mathrm{CE}=$ contrast enhanced image regions. $\mathrm{GC}=$ gamma corrected image regions. HE = histogram equalized image regions. R-90, R-180, $\mathrm{R}-270=90^{\circ}-, 180^{\circ}-, 270^{\circ}$-rotated image regions.

\begin{tabular}{llrrrrrrr}
\hline \hline \multirow{2}{*}{ Images } & \multirow{2}{*}{ Approach } & \multicolumn{7}{c}{ Classification Accuracy (\%) } \\
\cline { 3 - 9 } & & O & CE & GC & HE & R-90 & R-180 & R-270 \\
\hline \multirow{2}{*}{ Test-1 } & Ranklets+SVM & 98.96 & 98.96 & 98.96 & 98.96 & 98.96 & 98.96 & 98.96 \\
& Ridgelets+Dist & 96.79 & - & - & - & - & - & - \\
\hline \multirow{2}{*}{ Test-2 } & Ranklets+SVM & 99.38 & 99.23 & 99.23 & 99.23 & 99.38 & 99.38 & 99.38 \\
& Wavelets+Dist & 99.52 & - & - & - & 95.19 & 100.00 & 95.67 \\
\hline \multirow{2}{*}{ Test-3 } & Ranklets+SVM & 98.59 & 98.59 & 98.59 & 98.59 & 98.59 & 98.59 & 98.59 \\
& Wavelets+Dist & 93.55 & - & - & - & 83.67 & 100.00 & 83.47 \\
\hline \multirow{2}{*}{ Test-4 } & Ranklets+SVM & 100.00 & 100.00 & 100.00 & 100.00 & 100.00 & 100.00 & 100.00 \\
& Wavelets+SVM & 96.34 & - & - & - & - & - & - \\
\hline \hline
\end{tabular}

labeled with $\mathrm{CE}, \mathrm{GC}$, and $\mathrm{HE}$ to the case in which the LOO training phase is carried out on image regions extracted from the original images, whereas the testing phase on image regions extracted from the contrast-enhanced, gammacorrected, and histogram-equalized images; the columns labeled with R-90, R180 , and R-270 to the case in which the LOO training phase is carried out on image regions extracted from the original images, whereas the testing phase on image regions extracted from the $90^{\circ}-, 180^{\circ}-$, and $270^{\circ}$-rotated images, respectively.

By looking at the first row of Tab. 1, it is possible to notice that the LOO percentage classification accuracy reached on Test-1 is particularly high, i.e., $475 / 480=98.96 \%$ of image regions correctly classified. Also, this result is maintained perfectly unaltered for the gray-scale transformed and $90^{\circ}$-rotated versions of the image regions, meaning that the proposed texture features are insensitive to linear/non-linear monotonic gray-scale transformations and $90^{\circ}$ rotations of the original images.

High results are achieved also for Test-2, see the third row of Tab. 1. Here, the LOO percentage classification accuracy reaches $646 / 650=99.38 \%$ for the original and $90^{\circ}$-rotated images, whereas $645 / 650=99.23 \%$ for the grayscale transformed ones. Despite the surplus error for the gray-scale transformed images, the proposed texture features demonstrate to be invariant to linear/non-linear monotonic gray-scale transformations and $90^{\circ}$-rotations also in this case.

A perfect agreement among the results achieved on the original, gray-scale transformed, and $90^{\circ}$-rotated images is found again when evaluating the pro- 
posed texture features on Test-3, see the fifth row of Tab. 1 . Reaching 489/496= $98.59 \%$ of image regions correctly classified, the LOO percentage classification accuracy confirms the high results achieved in the previous cases.

Finally, no errors are made on the original, gray-scale transformed, and $90^{\circ}$ rotated images of Test-4, see the seventh row of Tab. 1. In this case, the LOO percentage classification accuracy scores $750 / 750=100.00 \%$.

\subsection{Comparisons}

For the purpose of comparison, the described results are compared with those achieved by three recent automatic texture classification systems found in literature and having being evaluated on the same Brodatz/Vistex original images, see Tab. 1. In particular, comparison is performed among automatic texture classification systems intended as a whole, i.e., as a set of specific features in combination with a specific classification technique, rather than singularly among the sets of features or the classification techniques adopted. First, Arivazhagan et al. (2006) proposed a texture classification approach based on ridgelet transform and minimum distance classification (i.e., Ridgelets+Dist). On a number of image regions extracted from the same 30 VisTex images used in Test-1, they achieved $96.79 \%$ of accuracy. As evident from the first and second rows of Tab. 1, Ranklets+SVM reaches higher results, i.e., $98.96 \%)$.

Second, Muneeswaran et al. (2005) developed a rotation-invariant approach based on wavelet transform and minimum distance classification (i.e., Wavelets+Dist). Evaluation was carried out on a number of image regions extracted from the same 26 Brodatz and 31 VisTex images used in Test-2 and Test-3. On the former, they found $99.52 \%, 95.19 \%, 100.00 \%$, and $95.67 \%$ of accuracy for the original and $90^{\circ}$-rotated images, respectively; similarly, they found $93.55 \%$, $83.67 \%, 100.00 \%$, and $83.47 \%$ on the latter, see the fourth and sixth rows of Tab. 1. As before, Ranklets+SVM performs generally better (i.e., $99.38 \%$ on the 26 Brodatz images and $98.59 \%$ on the 31 VisTex images); also, the results on the $90^{\circ}$-rotated images seem to be much more stable.

Finally, Li et al. (2003) used wavelet transform and SVM (i.e., Wavelets+SVM) on a number of image regions extracted from the same 30 Brodatz images used in Test-4.As evident from the seventh and eighth rows of Tab. 1, they reached $96.34 \%$ of accuracy versus the $100.00 \%$ achieved by Ranklets+SVM. Also, as the classification technique adopted by these two approaches is the same (i.e., SVM), a direct comparison among ranklet and wavelet features is allowed as well; in particular, owing to the superior results achieved by Ranklets+SVM, ranklet features seem to be more appropriate than wavelet features in solving the proposed texture classification problem. 


\section{Conclusions}

In this work, texture classification invariant to linear/non-linear monotonic gray-scale transformations and $90^{\circ}$-rotations is achieved by: (1) submitting each image to the ranklet transform; (2) extracting a number of texture features from the resulting ranklet images; (3) averaging, for each resolution, correspondent vertical, horizontal, and diagonal texture features; (4) assigning a texture class membership to the final texture feature vector by means of an SVM classifier.

On image regions extracted from four VisTex and Brodatz image datasets, classification results perform very well and demonstrate to be superior to those reached on the same image datasets by three recent texture classification methods found in literature (Arivazhagan et al., 2006; Muneeswaran et al., 2005; Li et al., 2003). More specifically, the LOO percentage classification accuracy achieves $98.96 \%$ on the original 30 VisTex images of Test-1, $99.38 \%$ on the original 26 Brodatz images of Test-2, $98.59 \%$ on the original 31 VisTex images of Test-3, and $100.00 \%$ on the original 30 Brodatz images of Test-4. Also, invariance to linear/non-linear monotonic gray-scale transformations and $90^{\circ}$-rotations is evidenced, as the above classification results are confirmed when the original images are subjected to contrast enhancement, gamma correction, histogram equalization, or $90^{\circ}$-rotation.

Owing to its capability of reaching high classification results, together with its robustness to illumination and viewpoint variations, this method could surely represent an attractive tool to address texture classification problems with such requirements.

\section{Acknowledgement}

The authors are grateful to Dr. Nico Lanconelli for fundamental comments and suggestions.

\section{References}

Arivazhagan, S., Ganesan, L., Subash Kumar, T. G., 2006. Texture classification using ridgelet transform. Pattern Recognition Letters 27 (16), 18751883.

Brodatz, P., 1966. Textures: a photographic album for artists and designers. Dover, Paris.

Campanini, R., Angelini, E., Iampieri, E., Lanconelli, N., Masotti, M., Roffilli, M., 2006. A ranklet-based CAD for digital mammography. In: Proceedings of the 8th International Workshop on Digital Mammography, Manchester, UK. pp. 340-346.

Charalampidis, D., Kasparis, T., 2002. Wavelet-based rotational invariant 
roughness features for texture classification and segmentation. IEEE Transactions on Image Processing 11 (8), 825-837.

Chen, J. L., Kundu, A., 1994. Rotation and gray scale transform invariant texture identification using wavelet decomposition and hidden Markov model. IEEE Transactions on Pattern Analysis and Machine Intelligence 16 (2), 208-214.

Chetverikov, D., 1982. Experiments in the rotation-invariant texture discrimination using anisotropy features. In: Proceedings of the 6th International Conference on Pattern Recognition. pp. 1071-1073.

Davis, L. S., Johns, S. A., Aggarwal, J. K., 1979. Texture analysis using generalized cooccurrence matrices. IEEE Transactions on Pattern Analysis and Machine Intelligence 1 (3), 251-259.

Efron, B., Tibshirani, R. J., 1994. An Introduction to the Bootstrap. Chapman \& Hall, London.

Gonzalez, R. C., Woods, R. E., 1992. Digital Image Processing, 3rd Edition. Addison-Wesley, Reading.

Idrissa, M., Acheroy, M., 2002. Texture classification using Gabor filters. Pattern Recognition Letters 23 (9), 1095-1102.

Li, S., Kwok, J. T., Zhu, H., Wang, Y., 2003. Texture classification using the support vector machines. Pattern Recognition 36 (12), 2883-2893.

Mallat, S., 1989. A theory for multiresolution signal decomposition: The wavelet representation. IEEE Transactions on Pattern Analysis and Machine Intelligence 11 (7), 674-693.

Manthalkar, R., Biswas, P. K., Chatterji, B. N., 2003a. Rotation and scale invariant texture features using discrete wavelet packet transform. Pattern Recognition Letters 24 (14), 2455-2462.

Manthalkar, R., Biswas, P. K., Chatterji, B. N., 2003b. Rotation invariant texture classification using even symmetric Gabor filters. Pattern Recognition Letters 24 (12), 2061-2068.

Masotti, M., 2006a. Exploring ranklets performances in mammographic mass classification using recursive feature elimination. In: Proceedings of the 16th IEEE International Workshop on Machine Learning for Signal Processing, Maynooth, Ireland. pp. 265-270.

Masotti, M., 2006b. A ranklet-based image representation for mass classification in digital mammograms. Medical Physics 33 (10), 3951-3961.

MIT Media Lab, 1995. VisTex: Vision texture image database. http://vismod.media.mit.edu/vismod/imagery/VisionTexture/.

Muneeswaran, K., Ganesan, L., Arumugam, S., Ruba Soundar, K., 2005. Texture classification with combined rotation and scale invariant wavelet features. Pattern Recognition 38 (10), 1495-1506.

Ojala, T., Pietikäinen, M., Mäenpää, T., 2002. Multiresolution gray-scale and rotation invariant texture classification with local binary patterns. IEEE Transactions on Pattern Analysis and Machine Intelligence 24 (7), 971-987.

Partio, M., Cramariuc, B., Gabbouj, M., 2004. Texture retrieval using ordinal co-occurrence features. In: Proceedings of the 6th Nordic Signal Processing 
Symposium, Espoo, Finland. Vol. 46. pp. 308-311.

Pichler, O., Teuner, A., Hosticka, B. J., 1996. A comparison of texture feature extraction using adaptive Gabor filtering, pyramidal and tree-structured wavelet transforms. Pattern Recognition 29 (5), 733-742.

Porter, R., Canagarajah, N., 1997. Robust rotation-invariant texture classification: Wavelet, Gabor filter and GMRF based schemes. In: IEE Proceedings: Vision, Image and Signal Processing. Vol. 144. pp. 180-188.

Smeraldi, F., 2002. Ranklets: orientation selective non-parametric features applied to face detection. In: Proceedings of the 16th International Conference on Pattern Recognition, Quebec City, Canada. Vol. 3. pp. 379-382.

Sukanya, P., Takamatsu, R., Sato, M., 2000. Multiscale image analysis through the surface-shape operator. Journal of Electronic Imaging 9 (3), 305-316.

Vapnik, V., 1995. The nature of statistical learning theory. Springer, New York.

Vapnik, V., 1998. Statistical learning theory. Wiley, New York.

Viola, P., Jones, M. J., 2004. Robust real-time face detection. International Journal of Computer Vision 57 (2), 137-154.

Weston, J., Watkins, C., 1998. Multi-class support vector machines. Technical Report CSD-TR-98-04, Department of Computer Science, Royal Holloway, University of London, Egham, UK.

Wu, W. R., Wei, S. C., 1996. Rotation and gray-scale transform-invariant texture classification using spiral resampling, subband decomposition and hidden Markov model. IEEE Transactions on Image Processing 5 (10), 14231434.

Xu, R., Zhao, X., Li, X., Chang, C. I., 2006. Target detection with improved image texture feature coding method and support vector machine. International Journal of Intelligent Technology 1 (1), 47-56. 